\title{
Characterization of Prototype Superconducting Magnetic Quadrupoles for the High Current Transport Experiment
}

\section{February 2001}

\author{
Steven M. Lund [SMLund@bl.gov, (510) 486-6936], GianLuca Sabbi \\ [GLSabbi@lbl.gov, (510) 495-2250], and Peter Seidl [PASeidl@lbl.gov, (510) 486-7653]
}

\section{Introduction}

Later phases of the High Current Transport Experiment (HCX) at LBNL, will employ superconducting magnetic quadrupole lenses to focus an intense, heavy-ion beam over approximately 50 lattice periods (100 quadrupoles). Here we present a characterization of a baseline quadrupole design suitable for transporting a single, low-energy $(\sim 2 \mathrm{MeV})$, high-current $(\sim 800 \mathrm{~mA})$ heavy-ion $\left(\mathrm{K}^{+}\right)$beam that will be provided from an existing injector and beam matching section . For optimal performance in this application, a compact quadrupole magnet providing high focusing strength and high field quality is required. The reference parameters that we have chosen take into account magnet development work by AML, LLNL, and MIT and result in a transport lattice well matched to programmatic needs with a lattice period of approximately $50 \mathrm{~cm}$. The goal of this note is to introduce a common framework where the magnetic performance of different designs can be compared. In that regard, we try to avoid the details of an earlier parameter note [1] where provisions for tweaks in magnet excitation, cryostat assembly, etc. were discussed in fairly general terms. This note is not intended to be a final specification for the HCX quadrupoles to be constructed or to be the sole basis on which competing magnet designs will be compared. Other aspects such as prototype test results, economic considerations, and attractiveness within the context of ultimate applications in multi-beam drivers for heavy-ion fusion (i.e., compatibility with magnet arrays, etc.) will all factor in the selection of the appropriate design option.

This note is organized as follows. Magnet characterizations including geometric and conductor parameters are given in Sec $\Pi$. Performance parameters to be reported that quantify the magnet properties are outlined in Sec III. Supporting information is included in appendices. A reference coordinate system to be employed in field calculations is defined in Appendix A. Detailed descriptions of the methods to be used to calculate the integrated field gradient and field errors of the magnet are given in Appendix B. Finally, in Appendix C, the magnet operating point is defined. 


\section{Design Parameters}

We specify geometric parameters and conductor properties of the magnet and ask that the integrated quadrupole gradient (i.e., the focusing strength) of the magnet be maximized beyond a minimum acceptable value while achieving optimal integrated field quality. Compact designs that achieve a high integrated gradient with good field quality will be judged as having superior performance. To simplify comparisons and the application of design results, we specify a reference coordinate system to be used in Appendix A and methods to calculate field quantities in Appendix B.

\section{Axial Geometry}

- Fixed axial length $\ell_{\text {cail }}=125 \mathrm{~mm}$ for the coil assembly. This will be measured from the extreme axial excursions of the current carrying turns of the coil assembly, excluding leads.

- Minimize axial length $\ell_{\text {mat }} \leq 155 \mathrm{~mm}$ of the magnet assembly. This will be measured from the extreme axial excursions of the full magnet assembly including coil forms, the iron yoke, leads and splices and their mechanical support elements.

\section{Transverse Geometry}

- Fixed clear bore radius $r_{\text {clear }}=35 \mathrm{~mm}$ for the magnet assembly. This is the intrinsic clear aperture of the magnet structure without auxiliary components (e.g. beam pipe and insulation for the warm bore beam pipe) and corresponds to the closest radial approach of materials to the axis of symmetry of the magnet.

- Maximum outer half-width $w_{\max }$ of the magnet assembly subject to $w_{\max } \leq 64 \mathrm{~mm}$. This width is measured along the axis of the magnetic pole as sketched in Figure 1 for rectangular and cylindrical magnet cross-sections. The limiting value of $w_{\max }=64 \mathrm{~mm}$ does not include the iron yoke and applies to the coil axial section only (i.e., for $|z| \leq \ell_{\text {coil }} / 2=62.5 \mathrm{~mm}$ ). No limit is presently set for the maximum outer extent of the lead/splice section, but this section should be made as compact as possible. 
Figure 1: The clear bore radius $r_{\text {clear }}$ and the maximum half-width $w_{\max }$ are illustrated in cross-section for magnets with cylindrical shell and rectangular coil geometry.

\section{Cylindrical}

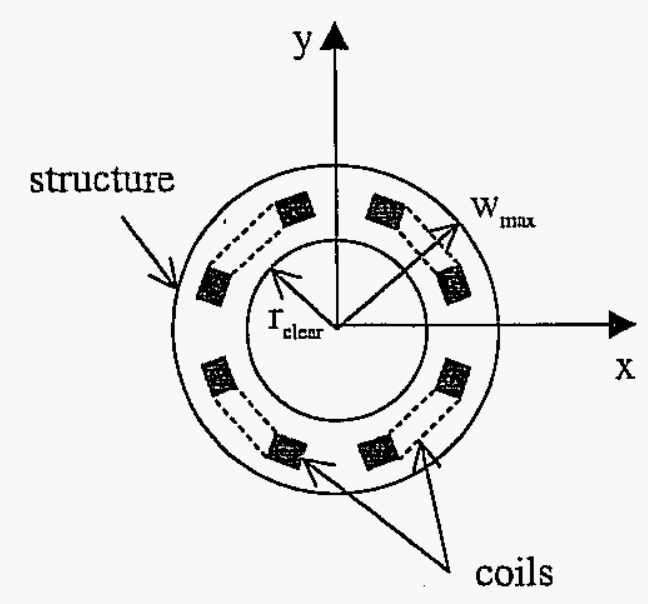

Rectangular

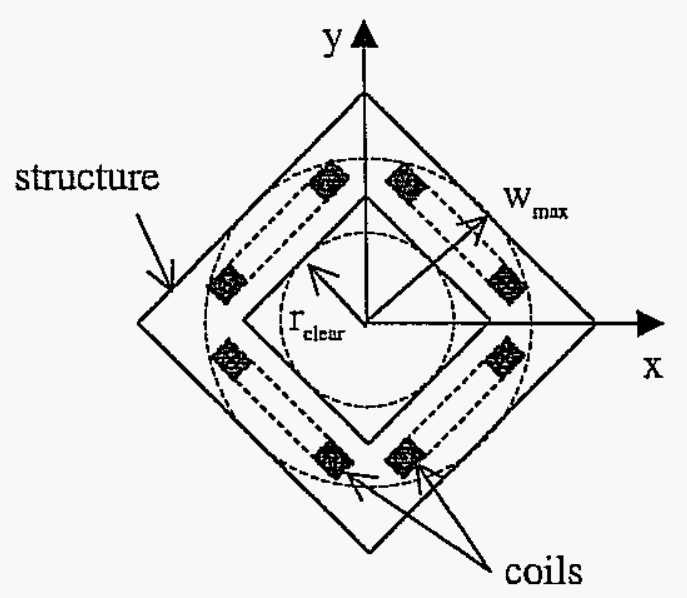

\section{Conductor Properties}

- In the range of interest (4-6 T), the dependence of the critical current density on field for $\mathrm{NbTi}$ conductor at $4.5 \mathrm{~K}$ is well approximated by a linear function:

$$
J_{c}\left(B, T_{0}\right)=J_{c}\left(B_{0}, T_{0}\right)+\frac{d I_{c}}{d B}\left(B_{0}, T_{0}\right) \cdot\left(B-B_{0}\right)
$$

This characterization along with the following parameters (close to "SSC" specifications) are to be assumed in calculating the minimum quench current of the magnet:

$$
\begin{aligned}
& B_{0}=5 \mathrm{~T} \text { (reference field) } \\
& T_{0}=4.5 \mathrm{~K} \text { (reference temperature) } \\
& J_{\mathrm{r}}\left(B_{0}, T_{0}\right)=2.55 \mathrm{kA} / \mathrm{mm}^{2} \\
& \frac{d J_{c}}{d B}\left(B_{0}, T_{0}\right)=-0.6 \mathrm{kA} /\left(\mathrm{mm}^{2}\right. \text { Tesla) }
\end{aligned}
$$

- The copper to superconductor ratio should be adjusted to obtain a copper current density $J_{e u} \leq 1.3 \mathrm{kA} / \mathrm{mm}^{2}$ at the short sample current (see Appendix C). 


\section{Integrated Quadrupole Gradient}

- Maximize the integrated quadrupole gradient $\int_{-\infty}^{\infty} B_{q}^{\prime} d z$ subject to $\int_{-\infty}^{\infty} B_{q}^{\prime} d z \geq 8.5$

Tesla. The gradient $\int_{-\infty}^{\infty} B_{q}^{\prime} d z$ should be calculated as specified in Appendix B at a nominal (operating) current excitation corresponding to $85 \%$ of the quench current (i.e., $I_{o p}=0.85 \cdot I_{s s}$, where $I_{s s}$ is the "short-sample" current as defined in Appendix C).

\section{Fieid Quality}

- Achieve the highest axially integrated field quality possible in terms of the fractional total field error $\delta F$ defined in Appendix B subject to $\delta F \leq 50\left(10^{-4}\right.$ units) measured at a good field radius of $r_{g}=25 \mathrm{~mm} \approx 0.71 \cdot r_{\text {clear }}$. This field error should include systematic contributions intrinsic to magnet, including leads, but not construction errors. The criteria $\delta F \leq 50$ should be taken as a maximum acceptable field error and efforts should be made to make $\delta F$ as small as possible.

Note:

Neither of the AML/LLNL first prototypes are fully consistent with the design characterizations given here. We require that future design iterations comply with these guidelines. However, the magnetic performance characterization proposed in Sec. III should be applied to present prototype designs with minimal changes before proceeding to a fully optimized design consistent with the requirements presented here. We believe that despite differences in the prototypes, meaningful characterizations of the first prototypes can be made with the information requested.

\section{Performance parameters}

The following data should be presented to characterize the magnet design:

Integrated Quadrupole Gradient $\int_{-\infty}^{\infty} B_{q}^{\prime} d z$ :

- Provide the integrated quadrupole gradient $\int_{-\infty}^{\infty} B_{q}^{\prime} d z$ of the magnet, calculated as defined in Appendix B at the operating point specified in Appendix C. 
- The value of the short sample current $I_{s s}$ and the cable parameters are also requested for reference purposes.

If the designers feel that the reference conductor properties result in a conservative estimate of the maximum quench current, an additional set of conductor properties (both superconductor and $J_{c u}$ ) can be analyzed. The corresponding short sample current and integrated gradient at the operating point should be provided.

\section{Field Quality}

a) Design Geometry:

- Tabulate both the fractional multipole errors $\delta b_{n}$ and $\delta a_{n}$, and the fractional total field error $\delta F$ at a reference radius $r_{g}=25 \mathrm{~mm} \approx 0.71 \cdot r_{\text {clear }}$. These integrated field errors are defined in Appendix B.

- Tabulate field errors at full and $50 \%$ coil excitation $\left(I=0.5 \cdot I_{o p}\right.$, with $\left.I_{o p}=0.85 \cdot I_{s s}\right)$.

Multipole errors should be given up to high enough harmonic number $n$ to demonstrate clear convergence for harmonics allowed by quadrupole symmetry $(n=2,6,10, \cdots)$ and any additional harmonics allowed by lead configurations, etc. We request field errors at full and 50\% coil excitation to evaluate possible changes due to iron saturation effects. Data on fractional multipole errors $\delta b_{n}$ and $\delta a_{n}$ are requested for completeness, but the fractional total field error $\delta F$ is the relevant quantity that the magnet geometry should be optimized with respect to. However, in most designs, it is reasonable to expect that the cascade in multipole error terms should converge rapidly and the terms should bear a clear relation on to the total field error $\delta F$.

The use of these integrated field errors allows the design of magnets with compensating errors in the ends and axial mid-section of the coils to achieve high average field quality. Simulations have determined that this should be sufficient for our purposes and any design with sufficient parametric freedom in the placement of coils and surfaces should be capable of achieving high integrated field quality. However, it is better if end errors can be smaller so that large compensations are not necessary.

\section{b) Construction Errors}

- Tabulate integrated fractional multipole field errors $\delta b_{n}$ and $\delta a_{n}$ that result from deviations from design geometry and design material properties, at the same good field radius used for the intrinsic field quality at nominal coil excitation ( $I=I_{o p}$ ). 
Assumptions and methods used in the analysis must be carefully specified to allow interpretation of results. Multipoles that are not allowed by the ideal magnet geometry should be presented along with any appreciable changes in the allowed, intrinsic harmonics. An example of methods to describe and report construction errors can be found in Ref. [2].

\section{Magnet Effective Length}

- Magnet effective axial length as defined by $\ell_{e f f}=\int_{-\infty}^{\infty} B_{q}^{\prime}(z) d z / B_{q}^{\prime}(z=0)$.

- Half gradient axial length $\ell_{1 / 2}$ where $B_{q}^{\prime}\left(z=\frac{\ell_{1 / 2}}{2}\right)=\frac{B_{q}^{\prime}(z=0)}{2}$.

- Calculate the effective lengths $\ell_{e f f}$ and $\ell_{1 / 2}$ at the magnet operating point $I=I_{o p}$ specified in Appendix C.

Here, $B_{q}^{\prime}(z)=\partial B_{x}(\vec{x}) /\left.\partial y\right|_{z=0, r=0}=\partial B_{y}(\vec{x}) /\left.\partial x\right|_{z=0, r=0}$ is the quadrupole gradient of the magnet (with the transverse $x$ - and $y$-coordinate axes chosen along the principle axes of the quadrupole field as specified in Appendix A). The parameters $\ell_{\text {eff }}$ and $\ell_{1 / 2}$ together help better characterize the fringe field properties of the magnet and the resulting optical properties. Smaller $\ell_{1 / 2}$ indicates a lesser extent of the axial fringe field.

\section{Axial and Transverse Geometric Factors}

- Axial geometric extent of the magnet assembly, $\ell_{\operatorname{mat}}$. Two values for $\ell_{\text {mat }}$ are requested, one measured from the axial ends of the coil forms excluding splices and leads and their mechanical support structures, and the other for the full axial length of the magnet including all splices, coil forms, leads, mechanical supports, etc.

- Radial geometric extents of the magnet assembly $w_{\max }$. Two values for $w_{\max }^{\prime}$ are requested, one for the coil axial section (i.e., for $|z| \leq \ell_{\text {coil }} / 2=62.5 \mathrm{~mm}$ ), and the other including the outermost extent of the lead/splice sections.

Achieving large integrated gradient $\int_{-\infty}^{\infty} B_{q}^{\prime} d z$ with smaller radial build $w_{m a t}$ demonstrates more effective use of conductor and materials. Smaller axial build $\ell_{m a t}$ demonstrates more efficient termination of axial magnet support structures, splices, etc. 


\section{References}

[1] Steven M. Lund, Peter Seidl, and Roger Bangerter, "Superconducting Quadrupole Magnet Parameters for the LBNL. High-Current Experiment," LBNL Internal Note, 20, December 1999.

[2] G.L. Sabbi, "HGQ_9711 Field Quality Table," Fermi National Accelerator Laboratory Publication TD-97-050, 14, November 1997.

[3] P.J. Lee, A Squitieri, B. Starch, W. Gabr-Rayan, C. Fischer, D. Vernon, R. Werner, and D. Larbalestier, "Development of Superconducting Strand for Low Beta Quadrupoles," FNAL, P.O. No. B94240, undated.

[4] Steven M. Lund, HIF Note Number 96-10, "On the 3D Field Structure of Rectangular Block Permanent Magnet Lenses," 7, September 1996. Section D of this paper contains a detailed, fully general discussion on the definition of 3D multipole moments.

[5] S. Caspi, M. Helm, and L. Laslett, "3D Field Harmonics," LBL Report SC-MAG-328, March 1991.

Y.

$\therefore$

$\therefore$ 


\section{Appendix A: Reference coordinate system}

To simplify comparisons and the evaluation of design data in beam dynamics evaluations, we require that the designers use the reference magnet orientation and coordinate system illustrated in Figure 2 below. The $z$-coordinate is taken along the axial symmetry axis of the magnet with $z=0$ corresponding to the center of the magnet straight section. The $z-$ axis is oriented from the magnet return end towards the lead end. The transverse $\mathrm{x}$-axis is horizontal and pointing to the right if an observer is facing the magnet from the lead end. The transverse $y$-axis is vertical and pointing upwards (i.e., a right-handed system with $\hat{x} \times \hat{y}=\hat{z}$ ). The magnet is oriented such that the magnetic midplanes of the principal quadrupole field component $\vec{B}_{q x}$ lie along the $\mathrm{x}$ and $\mathrm{y}$ axes while the magnetic poles are bisected by lines in the transverse $x-y$ plane that are at angles of $\pi / 4$ from the $x$ and $y$ coordinate axes, as sketched. The magnet powering convention is such to provide a current flow in the negative $\mathrm{z}$ direction in the octants which are next to the horizontal $(\mathrm{x})$ mid-plane, and in the positive $\mathrm{z}$ direction in the octants which are next to the vertical $(\mathrm{y})$ mid-plane. With this choice of coordinates, note that the principal quadrupole field $\vec{B}_{q x}$ satisfies $\partial B_{q y} / \partial x>0$.

Figure 2. Ideal magnet geometry and reference coordinates.

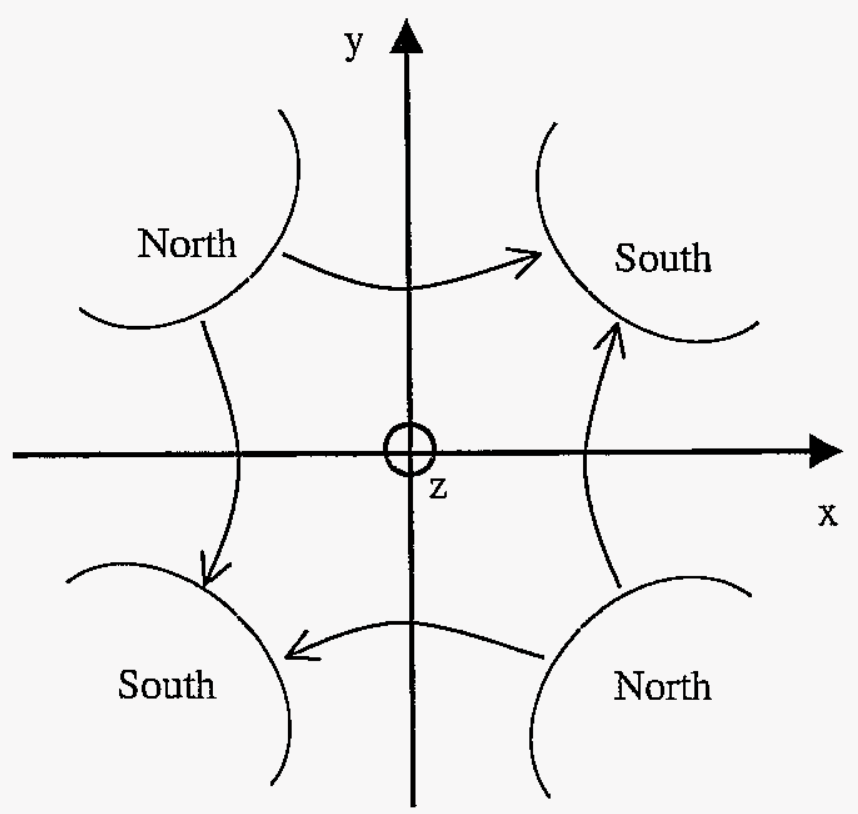




\section{Appendix B: Field calculation}

For the axially short magnets of interest, 3D multipole field data must be calculated with care to have meaning. Without proper resolution of field terms from pseudomoments resulting from the axial variation of the intrinsic (2D) multipoles [4-6], little direct meaning can be attached to the terms of an azimuthal harmonic decomposition of the field in an axial plane. To avoid this confusion, we wish the field quality data to be specified in terms of axial (z) integrals of the 3D magnetic field components. Denote integrated $\mathrm{x}$ - and $\mathrm{y}$-components of the $3 \mathrm{D}$ magnetic field $\vec{B}(x, y, z)$ by

$$
\begin{aligned}
& \hat{B}_{x}(x, y)=\int_{-\infty}^{\infty} B_{x}(x, y, z) d z \\
& \hat{B}_{y}(x, y)=\int_{-\infty}^{\infty} B_{y}(x, y, z) d z
\end{aligned}
$$

These 2D field components have dimension Tesla-meters and can be combined into a single complex $2 \mathrm{D}$ field $\hat{B}_{y}+i \hat{B}_{x}$ that is conveniently expanded within the clear magnet aperture as

$$
\bar{B}^{*}=\hat{B}_{y}+i \hat{B}_{x}=\sum_{n=1}^{\infty}\left(B_{n}+i A_{n}\right)\left(\frac{\bar{z}}{r_{0}}\right)^{n-1}=\sum_{n=1}^{\infty} \bar{C}_{n}\left(\frac{\bar{z}}{r_{0}}\right)^{n-1}
$$

Here, over-bars denote complex quantities, $i=\sqrt{-1}, \bar{z}=x+i y, r_{0}$ is a (arbitrary for purposes here) normalization radius, $n$ is a harmonic index, and the $\bar{C}_{n}=B_{n}+i A_{n}$ are complex multipole coefficients with real "normal" and "skew" components $B_{n}$ and $A_{n}$. Note that the $n=1$ harmonics describe the dipole field components, $n=2$ the quadrupole field components, $n=3$ the sextupole components, etc. For a magnet geometry with intrinsic quadrupole symmetry, only non-skew (e.g., all $A_{n}=0$ and the $B_{n}$ may be nonzero) harmonjes with $n=2,6,10, \cdots$ exist and $B_{2}$ will be positive for the choice of coordinates defined. To make physical connection with the integrated quadrupole gradient, which is an important measure of the focusing strength of the magnet, we denote

$$
\int B_{q}^{\prime} d z \equiv B_{2}
$$


In this notation, $B_{q}^{\prime}=\left.\frac{\partial B_{x}}{\partial y}\right|_{x=0, y=0}=\left.\frac{\partial B_{y^{\prime}}}{\partial x}\right|_{x=0, y=0}$ is the local, $z$-varying quadrupole gradient of the magnet, which is directly related to the integrated moment $B_{2}$ as specified.

Field quality should be measured in terms of fractional errors relative to the integrated quadrupole moment within a cylindrical good field radius of $r=\sqrt{x^{2}+y^{2}}=r_{g}$ contained within the clear magnet aperture. Both multipole error terms and total field errors will be requested at the good field radius as a fraction of the quadrupole field amplitude. First, we request fractional multipole errors:

$$
\delta b_{n}=\frac{B_{n}}{B_{2}}\left(\frac{r_{g}}{r_{0}}\right)^{n-2} \cdot 10^{4} \quad \delta a_{n}=\frac{A_{n}}{B_{2}}\left(\frac{r_{g}}{r_{0}}\right)^{n-2} \cdot 10^{4}
$$

These terms should be calculated till well converged for all multipoles intrinsic to quadrupole geometry $(n=6,10,14, \cdots)$ and for any additional multipoles $(n=1,3,5,7, \cdots)$ that may result from intrinsic geometric effects such as leads and coil imbalances and from construction errors such as surface imperfections, winding errors, and materials defects. Terms should be tabulated at each good field radius until clearly converged. This will generally require more terms for larger good field radii. Since particles respond to the total field and not individual multipoles, we also request a total field error

$$
\delta F=\frac{\operatorname{Max}_{\theta}\left|\bar{B}^{*}\left(r=r_{g}, \theta\right)-B_{2}\left(r_{g} / r_{0}\right) e^{i \theta}\right|}{B_{2}\left(r_{g} / r_{0}\right)} \cdot 10^{4}
$$

Here, $\operatorname{Max}_{\theta}$ denotes the maximum value in azimuthal angle. Note that the numerator of this expression corresponds to the maximum of the integrated field error (desired quadrupole term subtracted) around the azimuth of the circle defining the good field radius and the denominator is the (constant in $\theta$ ) magnitude of the integrated quadrupole field at the good field radius. This field error contains all multipole components and can only decrease when moving further within the circle defined by the good field radius since the integrated field components satisfy a $2 D$ vacuum Laplace equation within the magnet aperture. It should be demonstrated that this total field error occurs at a particular angle rather than assuming it must occur at $\theta=45^{\circ}$.

Some comments on the application of this formulation are in order. When calculating the harmonic coefficients $A_{n}$ and $B_{n}$, sufficient terms and data points (azimuthal nodes) must be taken to ensure accuracy. Care must be taken for larger good field radii $r_{g}$, because as $r_{g}$ approaches the clear bore of the magnet, the series expansion becomes nonconvergent and more, higher-order harmonics become necessary. The use of the normalization radius $r_{0}$ in the 2D-field expansion is also arbitrary and is employed so that 
all multipole terms $\bar{C}_{n}=B_{n}+i A_{n}$ can be conveniently measured in the same units. The quantities $B_{n} / r_{0}{ }^{n-1}$ and $A_{n} / r_{0}{ }^{n-1}$ will be independent of the specific choice of $r_{0}$.

\section{Appendix C: Operating point}

The value of the integrated gradient $\int B_{q}^{\prime} d z$ should be calculated with a coil excitation $I_{\text {op }}$ corresponding to $15 \%$ less current than the so-called "short-sample" current $I_{s s}$ defined as follows (see Figure 3 below). Plot the quench current $I_{q}$ of a sample of cable as function of the magnetic field $B$ applied to the sample. On the same axes, plot the current excitation $I$ of the 3D-magnet design versus the peak magnitude $B$ of the 3D magnetic field within the coil. The short sample current $I_{x s}$ is the current at the intercept of these two curves. The magnet operating point is defined at a current excitation of $I=I_{o p} \equiv 0.85 \cdot I_{s s}$. This margin is needed to ensure that production magnets can be operated at nominal gradient with little or no training. The $15 \%$ design margin taken may be adjusted based on prototype test results.

Figure 3. Short-sample current definition.

\section{I, Current}

Short Sample Current, $\mathrm{I}_{\mathrm{ss}}$

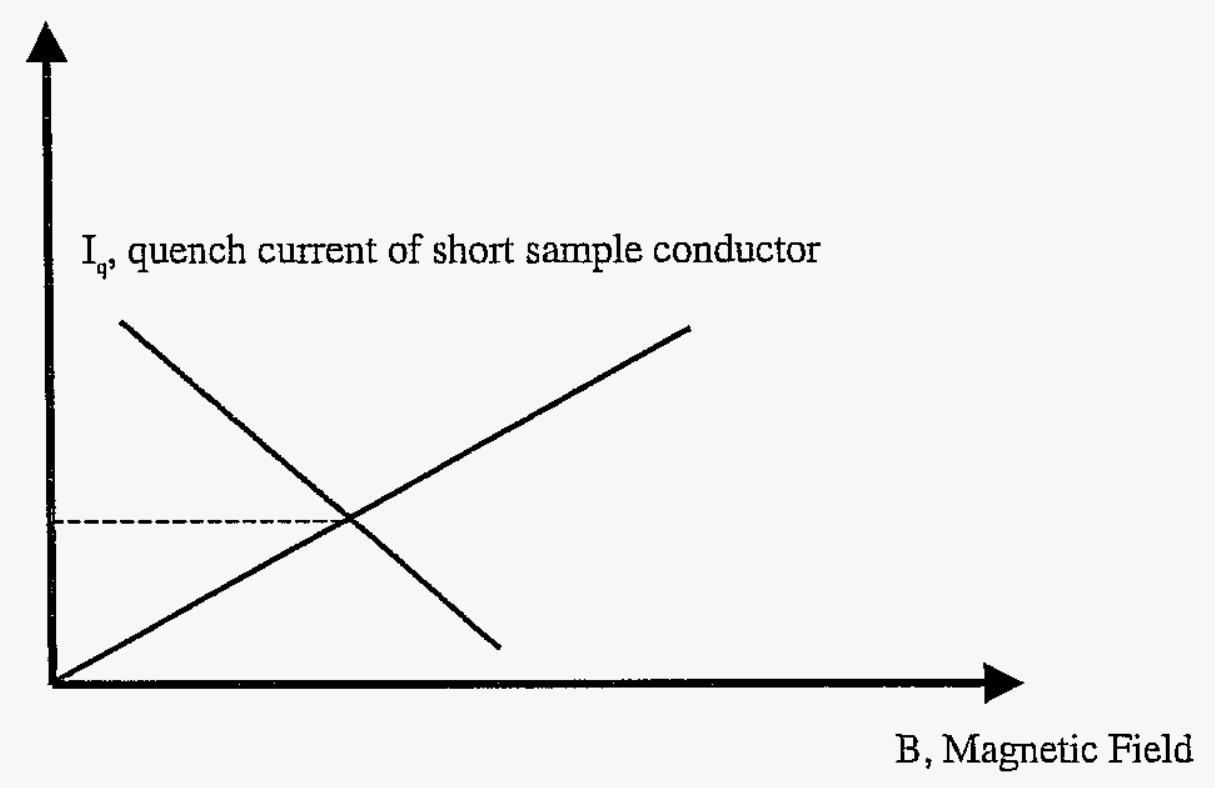

